\title{
Reductions in length of stay, narcotics use, and pain following implementation of an enhanced recovery after surgery program for 1- to 3-level lumbar fusion surgery
}

\author{
G. Damian Brusko, BS, ${ }^{1}$ John Paul G. Kolcun, BS, ${ }^{1}$ Julie A. Heger, BA, ${ }^{1}$ Allan D. Levi, MD, PhD, \\ Glen R. Manzano, MD, ${ }^{1}$ Karthik Madhavan, MD, ${ }^{1}$ Timur Urakov, MD, ${ }^{1}$ Richard H. Epstein, MD, ${ }^{2}$ and \\ Michael Y. Wang, MD'
}

Departments of ${ }^{1}$ Neurological Surgery and ${ }^{2}$ Anesthesiology, University of Miami Miller School of Medicine, Miami, Florida

OBJECTIVE Lumbar fusion is typically associated with high degrees of pain and immobility. The implementation of an enhanced recovery after surgery (ERAS) approach has been successful in speeding the recovery after other surgical procedures. In this paper, the authors examined the results of early implementation of ERAS for lumbar fusion.

METHODS Beginning in March 2018 at the authors' institution, all patients undergoing posterior, 1- to 3-level lumbar fusion surgery by any of 3 spine surgeons received an intraoperative injection of liposomal bupivacaine, immediate single postoperative infusion of 1-g intravenous acetaminophen, and daily postoperative visits from the authors' multidisciplinary ERAS care team. Non-English- or non-Spanish-speaking patients and those undergoing nonelective or staged procedures were excluded. Reviews of medical records were conducted for the ERAS cohort of 57 patients and a comparison group of 40 patients who underwent the same procedures during the 6 months before implementation.

RESULTS Groups did not differ significantly with regard to sex, age, or BMI (all $p>0.05$ ). Length of stay was significantly shorter in the ERAS cohort than in the control cohort ( 2.9 days vs 3.8 days; $p=0.01$ ). Patients in the ERAS group consumed significantly less oxycodone-acetaminophen than the controls on postoperative day (POD) 0 (408.0 mg vs $1094.7 \mathrm{mg} ; \mathrm{p}=0.0004)$, POD 1 (1320.0 mg vs $1708.4 \mathrm{mg} ; \mathrm{p}=0.04$ ), and POD 3 (1500.1 mg vs $2105.4 \mathrm{mg} ; p=0.03$ ). Postoperative pain scores recorded by the physical therapy and occupational therapy teams and nursing staff each day were lower in the ERAS cohort than in controls, with POD 1 achieving significance (4.2 vs $6.0 ; p=0.006)$. The total amount of meperidine ( $8.8 \mathrm{mg}$ vs $44.7 \mathrm{mg} ; \mathrm{p}=0.003$ ) consumed was also significantly decreased in the ERAS group, as was ondansetron (2.8 mg vs $6.0 \mathrm{mg} ; p=0.02$ ). Distance ambulated on each POD was farther in the ERAS cohort, with ambulation on POD 1 (109.4 ft vs $41.4 \mathrm{ft} ; p=0.002$ ) achieving significance.

CONCLUSIONS In this very initial implementation of the first phase of an ERAS program for short-segment lumbar fusion, the authors were able to demonstrate substantial positive effects on the early recovery process. Importantly, these effects were not surgeon-specific and could be generalized across surgeons with disparate technical predilections. The authors plan additional iterations to their ERAS protocols for continued quality improvements.

https://thejns.org/doi/abs/10.3171/2019.1.FOCUS18692

KEYWORDS enhanced recovery after surgery; ERAS; length of stay; cost; pain control; fast-track surgery; Exparel; lumbar fusion; outcomes; quality

$\mathrm{L}$ UMBAR spinal fusion can be highly successful in properly selected patients, and numerous studies have documented improvements in metrics designed to measure health outcomes and quality of life improvements following surgery. ${ }^{10}$ However, these procedures typically involve a lengthy recovery period that is associated with significant pain, immobility, and economic cost. The majority of patients will overcome this recovery period to resume their normal lives; however, the burden of surgical intervention remains significant.

The enhanced recovery after surgery (ERAS) movement began in Denmark as a practice concept integrating

ABBREVIATIONS ERAS = enhanced recovery after surgery; LOS = length of stay; MIS = minimally invasive surgery; POD = postoperative day; PT/OT = physical therapy and occupational therapy.

SUBMITTED December 1, 2018. ACCEPTED January 16, 2019

INCLUDE WHEN CITING DOI: 10.3171/2019.1.FOCUS18692. 
TABLE 1. Summary of the initial interventions for the ERAS program

\begin{tabular}{|c|c|c|}
\hline Stage/Intervention & Brief Description & Enhanced Recovery Principle \\
\hline \multicolumn{3}{|l|}{ Intraop } \\
\hline Liposomal bupivacaine injection & $\begin{array}{l}\text { Long-acting local anesthetic injection targeted directly to } \\
\text { areas of soft-tissue trauma }\end{array}$ & Minimize postop pain to facilitate early ambulation \\
\hline \multicolumn{3}{|l|}{ Postop } \\
\hline IV infusion of acetaminophen & $\begin{array}{l}\text { Immediate infusion of } 1 \mathrm{~g} \text { of acetaminophen beginning } \\
\text { while patient remains in OR }\end{array}$ & Minimize postop pain to facilitate early ambulation \\
\hline Daily visits from ERAS care team & $\begin{array}{l}\text { Daily visits to assess pain control, monitor ambulation } \\
\text { goals, \& assist w/ discharge planning }\end{array}$ & Facilitate discharge plan \& ensure patient satisfaction \\
\hline
\end{tabular}

$\mathrm{IV}=$ intravenous; $\mathrm{OR}=$ operating room.

multidisciplinary, perioperative care programs. Previously known as "fast-track surgery" or "enhanced recovery programs," ERAS became popularized in the 1990s to reduce the length of stay (LOS) after elective surgery through integrated approaches. ${ }^{3-7}$ Core ERAS tenets included 1) a focus on the patient's journey through surgery; 2) a multidisciplinary team approach; 3 ) interventions designed to reduce pain, morbidity, and recovery time; and 4) a data-driven iterative improvement process. The success of ERAS has been evidenced by the proliferation of programs across 6 continents as well as in most surgical specialties.

To date, there have been few peer-reviewed publications regarding spine ERAS programs that have actually been

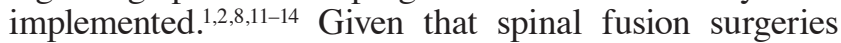
can be painful, morbid, and costly, the implementation of a spine ERAS program would be a major advancement in the field. In an effort to develop evidence-driven protocols for ERAS, we embarked on a quality improvement project to address the challenges spinal surgeons face in the postoperative period. This is distinct from our techniquedriven "awake spinal fusion" procedure that seeks to improve patient outcomes through alterations in surgical technique. ${ }^{14}$ This report summarizes our initial experience with the first iteration of our "version 1.0" program and its effects on the acute care period.

\section{Methods \\ ERAS Program Interventions}

The first iteration of our neurosurgical spine ERAS program began in March 2018 at the University of Miami Hospital. For the initial implementation, we chose to target postoperative pain and early ambulation as the drivers of enhanced recovery with 3 interventions (Table 1). First, patients received an intraoperative injection of $20 \mathrm{~mL}$ of liposomal bupivacaine (Exparel, Pacira Pharmaceuticals) mixed with $20 \mathrm{~mL}$ of bupivacaine hydrochloride. For 3-level fusions, dilution with $10-20 \mathrm{~mL}$ of injectable sterile saline was sometimes needed to achieve adequate volume for injection. In open procedures, the injection was administered during wound closure subfascially in a circumferential manner. During minimally invasive surgery (MIS), the liposomal bupivacaine mixture was injected along the percutaneous pedicle screw track prior to screw insertion. The injected amount was distributed equally around the single large incision for open cases or divided evenly between each of the percutaneous screw sites for MIS. Second, patients received a single postoperative infusion of $1 \mathrm{~g}$ of intravenous acetaminophen (Ofirmev, Mallinckrodt Pharmaceuticals). Medication administration was begun in the immediate postoperative period while patients remained in the operating room awaiting extubation and transport to the postanesthesia care unit (PACU). Infusion was subsequently completed either in the PACU or on the surgical hospital floor, and no other acetaminophen infusions were given during the remainder of the hospital stay. Patients did receive oxycodoneacetaminophen (5 mg-325 mg) tablets as needed for pain, as part of the institutional spine postoperative order set. However, this was not a change from our standard postoperative orders for all spine patients and, therefore, was not included as one of the 3 interventions. Finally, each patient received a daily postoperative visit from a member our ERAS care team (G.D.B., J.A.H., or J.P.K.) consisting of medical students working closely with the neurosurgical resident staffing the floor. The purpose of these visits, which occurred separately from daily neurosurgical rounds, was to ensure that patients had adequate pain management and were receiving early mobilization from the physical therapy and occupational therapy (PT/OT) teams, and to facilitate patient discharge plans, ensuring appropriate medical devices such as braces and walkers were delivered on time. If necessary, a member of the ERAS care team immediately notified the resident of any medical concerns requiring attention or the need to place orders for a patient.

\section{Patient Series}

All adult patients (18 years or older) who underwent elective, posterior, 1- to 3-level lumbar fusion surgery by one of 3 spine surgeons (M.Y.W., A.D.L., or G.R.M.) were enrolled in the ERAS program (Table 2). Additional inclusion criteria included both open and MIS fusion surgery. Surgeon preference regarding operative devices, techniques, and other technologies was not limited by the protocol, provided that addition of the 3 ERAS interventions was completed. All patients were English or Spanish speaking. Patients who underwent staged, multiday, or front/back procedures; emergency department-to-hospital admission; and 4 or more levels of fusion in the lumbosacral region or a fusion surgery of any level in the cervi- 
TABLE 2. Eligibility criteria for our ERAS program

\begin{tabular}{cc}
\hline \multicolumn{1}{c}{ Inclusion Criteria } & \multicolumn{1}{c}{ Exclusion Criteria } \\
\hline $\begin{array}{c}\text { Single surgeon (M.Y.W., A.D.L., } \\
\text { G.R.M.) }\end{array}$ & $\begin{array}{c}\text { Surgery w/ other or multiple } \\
\text { surgeons }\end{array}$ \\
\hline $\begin{array}{c}\text { Single institution (University of } \\
\text { Miami Hospital) }\end{array}$ & $\begin{array}{c}\text { Surgery at a different affiliated } \\
\text { hospital }\end{array}$ \\
\hline Elective surgery & ED-to-hospital admission \\
\hline Posterior-only lumbar fusion & Staged or multiday procedures \\
\hline Open \& MIS surgery & Front/back or lateral procedures \\
\hline 1-3 levels fused & $\geq 4$ levels fused \\
\hline Revision surgery & Cervical or thoracic surgery \\
\hline English- or Spanish-speaking & Non-English- or non-Spanish- \\
patient & speaking patient \\
\hline
\end{tabular}

$E D=$ emergency department.

cal or thoracic regions following program implementation were excluded.

A pre-ERAS comparison cohort of 40 patients who underwent the same procedures during the 6 months immediately preceding ERAS program implementation was used for analysis. The comparison-group patients would have met all inclusion criteria for enrollment in the ERAS program had the implementation process been in effect during the time. All patients in both groups underwent their operation and postoperative care entirely at the University of Miami Hospital.

All data were prospectively collected for the ERAS patients from electronic medical records. Data for the pre-ERAS group were collected based on a retrospective review of medical records prior to statistical analysis. $\mathrm{Pa}-$ tients were evaluated for baseline demographic characteristics, LOS stay, in-hospital medication consumption, and distance ambulated. Pain scores collected by nursing staff and from the PT/OT teams each day were also collected.

Statistical analysis of all data was conducted using Microsoft Excel data analysis tools and analyzed using a 2 -sample t-test assuming equal variances using an alpha value of 0.05 . A p value $<0.05$ was considered statistically significant.

\section{Results}

\section{Baseline Characteristics}

A total of 57 patients were enrolled in our ERAS program during the first 6 months of implementation. Clinical and demographic data can be found in Table 3. All patients in the ERAS group received each of the 3 interventions described in our protocol. The proportion of patients for each surgeon was similar between the ERAS (M.Y.W.: 65\%; G.R.M.: 21\%; A.D.L.: 14\%) and pre-ERAS (M.Y.W.: 70\%; G.R.M.: 15\%; A.D.L.: 15\%) cohorts. The average age $( \pm \mathrm{SD})$ of the ERAS cohort was $65.5 \pm 9.3$ years (range 48-89 years), compared with $68.1 \pm 9.9$ years (range $44-91$ years) in the pre-ERAS population $(\mathrm{p}=0.09)$. The male/ female ratios for the ERAS and pre-ERAS groups were $33: 24$ and 21:20, respectively $(\mathrm{p}=0.30)$. The average BMI of the ERAS patients $(29.1 \pm 5.4)$ was greater than that in
TABLE 3. Clinical and demographic data for ERAS and pre-ERAS cohorts

\begin{tabular}{ccc}
\hline Characteristic & Value & p Value \\
\hline Age, yrs & & 0.09 \\
\hline ERAS & $65.5 \pm 9.3(48-89)$ & \\
\hline Pre-ERAS & $68.1 \pm 9.9(44-91)$ & \\
\hline Male/female ratio & & \\
\hline ERAS & $33: 24$ & 0.09 \\
\hline Pre-ERAS & $21: 20$ & \\
\hline BMl & & \\
\hline ERAS & $29.1 \pm 5.4$ & \\
\hline Pre-ERAS & $27.6 \pm 5.6$ & \\
\hline Total levels fused & & \\
\hline ERAS & 92 & \\
\hline Pre-ERAS & 62 & $0.01^{*}$ \\
\hline Levels fused & & \\
\hline ERAS & $1.61 \pm 0.8$ & \\
\hline Pre-ERAS & $1.55 \pm 0.7$ & \\
\hline LOS, days & $2.9 \pm 1.9$ & \\
\hline ERAS & $3.8 \pm 1.8$ & \\
\hline Pre-ERAS &
\end{tabular}

Values are mean $\pm S D$ (range) unless indicated otherwise.

* Statistically significant.

the comparison group $(27.6 \pm 5.6)$, but the difference was not statistically significant $(\mathrm{p}=0.09)$. In total, 92 lumbar levels were fused in the ERAS group and 62 levels were fused in the control group. However, the average number of levels fused in both groups did not differ significantly (1.61 \pm 0.8 levels vs $1.55 \pm 0.7$ levels; $p=0.34)$.

\section{Narcotics Usage}

We analyzed narcotics consumption of several different pain medications used postoperatively on an as-needed basis in our spine program pain order set, which was not included as a standardized ERAS intervention in this initial iteration. We observed significant reductions in milligrams of oral oxycodone-acetaminophen consumption for the ERAS cohort compared with controls on postoperative day (POD) $0(408.0 \pm 527.2 \mathrm{mg}$ vs $1094.7 \pm 847.6 \mathrm{mg} ; \mathrm{p}$ $=0.0004)$, POD $1(1320.0 \pm 1026.4 \mathrm{mg}$ vs $1708.4 \pm 819.6$ $\mathrm{mg} ; \mathrm{p}=0.04)$, and POD $3(1500.1 \pm 778.5 \mathrm{mg}$ vs $2105.4 \pm$ $1090.6 \mathrm{mg} ; \mathrm{p}=0.03$ ), as well as during total hospital stay $(2729.5 \pm 4594.3 \mathrm{mg}$ vs $5230.3 \pm 3920.3 \mathrm{mg} ; \mathrm{p}=0.003)$ (Fig. 1). The average amount of meperidine consumed each POD did not achieve significance, but the total amount of meperidine consumed was significantly decreased in the ERAS group compared with the pre-ERAS group (8.8 \pm $32.9 \mathrm{mg}$ vs $44.7 \pm 87.5 \mathrm{mg} ; \mathrm{p}=0.003$ ). Furthermore, ERAS patients spent on average less time requiring intravenous pain medication (1.6 \pm 1.2 days) than the comparison cohort $(2.0 \pm 1.1$ days $)$ with the difference nearly achieving significance $(p=0.06)$. ERAS patients tended to consume more hydromorphone daily and overall than the pre-ERAS patients, but these results did not achieve significance (all $\mathrm{p}>0.1$. 


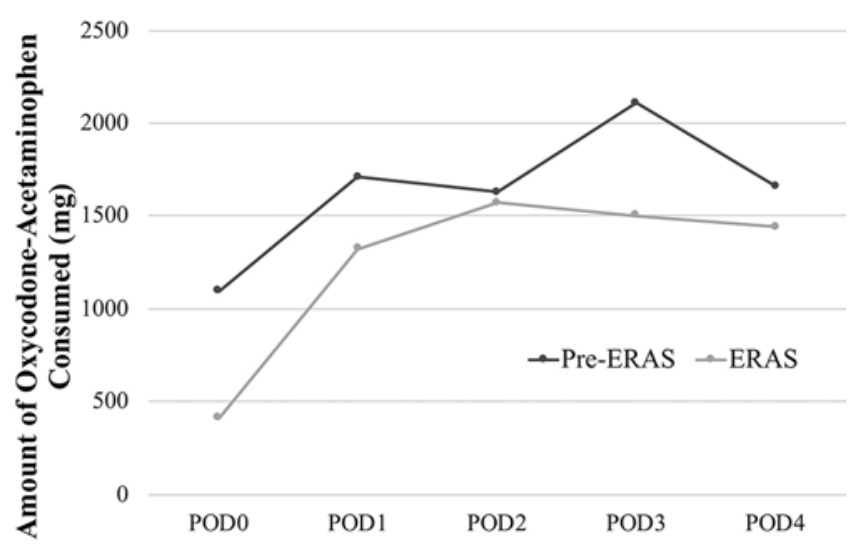

FIG. 1. Daily average oxycodone-acetaminophen consumption on each POD for ERAS and pre-ERAS groups.

\section{Antiemetics}

Ondansetron and promethazine are the 2 antiemetic drugs available in our spine program. The total amount of ondansetron required for the ERAS patients $(2.81 \pm$ $4.3 \mathrm{mg}$ ) was significantly less than that of the pre-ERAS group ( $6.0 \pm 10.5 \mathrm{mg} ; \mathrm{p}=0.02$ ) (Fig. 2). The total amount of promethazine consumed was slightly greater for the ERAS patients $(14.8 \pm 22.7 \mathrm{mg})$ than for the controls $(12.7$ $\pm 10.5 \mathrm{mg})$, but it was not significant $(\mathrm{p}=0.31)$.

\section{Ambulation}

The distance ambulated with the PT/OT teams each day was collected from their respective progress notes as a measure of early ambulation. The average distance ambulated was farther in the ERAS cohort on each POD, with POD $1(109.4 \pm 130.4 \mathrm{ft}$ vs $41.4 \pm 62.0 \mathrm{ft}$; $\mathrm{p}=0.002)$ achieving significance (Fig. 3). Total distance ambulated during the hospital stay was greater in the pre-ERAS patients $(448.8 \pm 431.2 \mathrm{ft})$ than in the ERAS patients (339.1 $\pm 371.6 \mathrm{ft} ; \mathrm{p}=0.09$ ). However, this may be the result of a

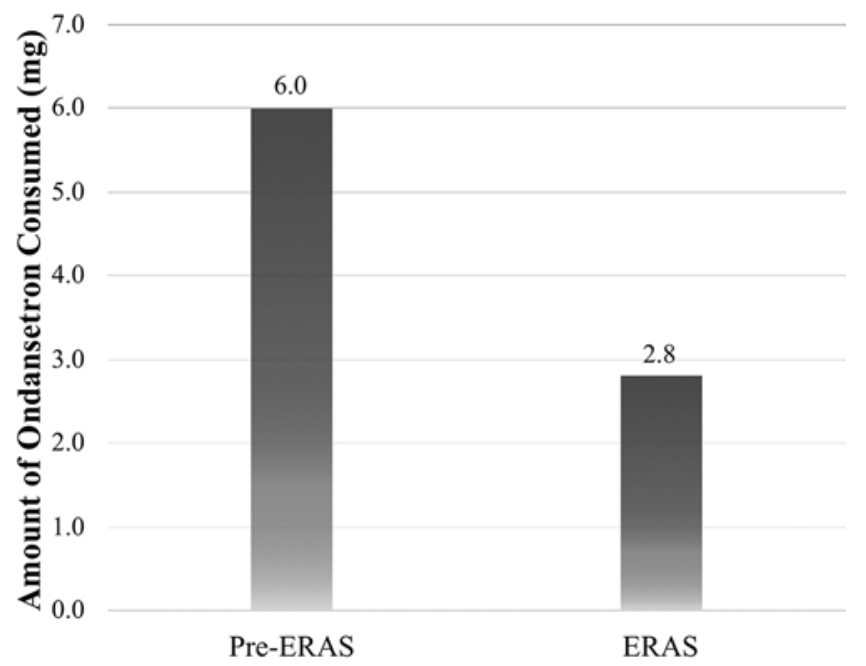

FIG. 2. Total ondansetron consumption during the hospital stay for ERAS and pre-ERAS groups.

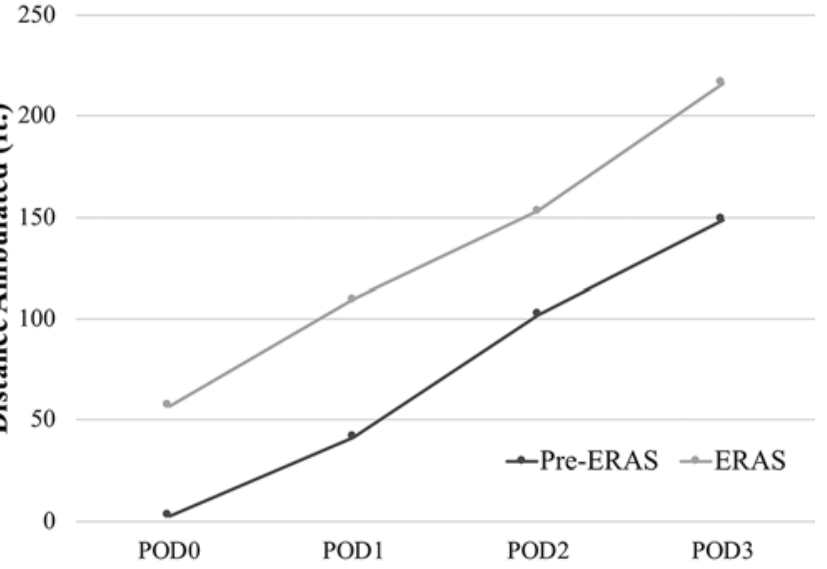

FIG. 3. Daily average distance ambulated with PT/OT teams on each POD for ERAS and pre-ERAS groups.

longer hospital stay, as total distance ambulated between PODs 0 and 3 was greater for the ERAS patients (300.5 \pm $404.0 \mathrm{ft})$ than pre-ERAS $(241.6 \pm 250.3 \mathrm{ft} ; \mathrm{p}=0.22)$.

\section{Pain}

We separately analyzed postoperative subjective pain scores rated on a scale of 1-10 recorded by both the PT/ OT teams and the nursing staff. Pain scores recorded by the PT/OT teams each postoperative day were lower in the ERAS cohort than in the control group, with POD 1 achieving significance $(4.2 \pm 3.2$ vs $6.0 \pm 3.2 ; \mathrm{p}=0.006)$ (Fig. 4). The increase in pain score on POD 2 for the ERAS patients may be attributable to the effect of the liposomal bupivacaine beginning to decline after 48-72 hours postinjection. Subjective pain scores reported by the floor nursing staff also demonstrated a trend of decreased pain on each POD but did not achieve any statistical significance.

\section{Length of Stay}

Patients in both cohorts had similar management by the nursing staff, social workers, and case management. There was no enforced discharging or "clinical pathway" treatment of patients, other than the daily rounds. Hospital LOS was significantly shorter in the ERAS cohort ( $2.9 \pm$ 1.9 days) than in the pre-ERAS cohort $(3.8 \pm 1.8$ days; $\mathrm{p}$ $=0.01$ ) (Fig. 5).

\section{Discussion}

The success of ERAS programs has been clear. However, to date, the majority of ERAS programs have focused on hollow viscus and thoraco-abdominal cavity surgeries. While the positive effects of ERAS have been well demonstrated, the postoperative challenges these subspecialties face differ somewhat from the challenges faced by spinal surgeons. For typical short-segment lumbar fusions for degenerative disease, the major challenge after surgery can be pain control and mobilization to prevent postoperative complications. This is more of an issue for spinal surgeons for the following reasons: 1) Most patients having surgery have already been in pain for prolonged periods of time, 


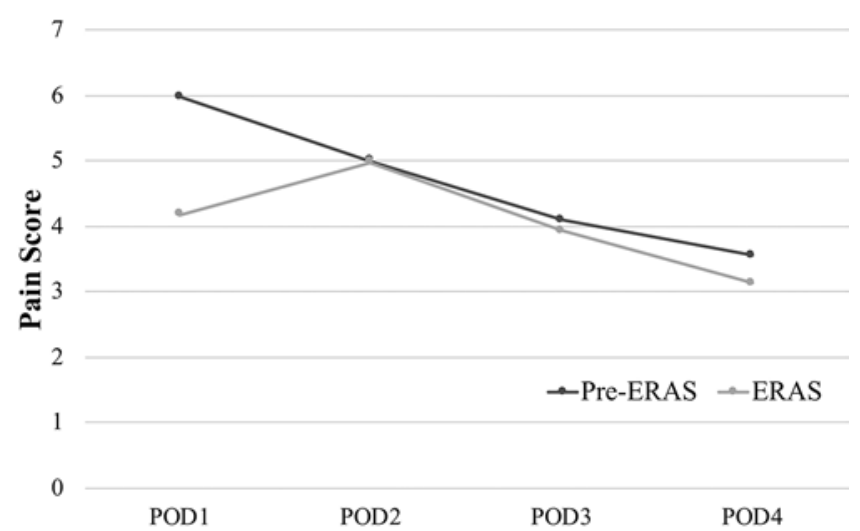

FIG. 4. Subjective postoperative pain scores recorded by PT/OT teams on each POD for ERAS and pre-ERAS groups.

so they arrive sensitized to pain triggers and often harbor pain-related behaviors. 2) Patients may be opiate dependent or resistant, and thus standard pain control regimens may be inadequate. 3) A major metric of success from the operation is pain relief, leading to fear early on that the operation has been unsuccessful. 4) Surgeries in the lumbar spine involve significant deep muscle tissue dissection and retraction, resulting in substantial pain. 5) The conflicting message of bracing and immobilization to allow for bony fusion has to be counterbalanced against mobilization to prevent complications. 6) Major variations in technique, practice, and outcomes lead to an inconsistent concept in the patient's mind of what to expect.

We sought to implement a simple and straightforward set of interventions designed to reduce the burden of recovery. The patient population and disease process we selected is among the most commonly encountered. Short-segment lumbar fusions are versatile for treating a spectrum of diseases, including nerve entrapment syndromes, segmental instability, and less severe deformities. Because these surgeries are common, the societal burden of prolonged recovery is high and the opportunity for impact is large. We also performed the study in a standard setting, which is a medium-sized (560-bed) hospital with equipment and capabilities similar to many community hospitals. The interventions employed also do not require highly specialized or complex technologies, facilities, or manpower. As such, they fulfill a basic tenet of successful ERAS programs, which is that they can be implemented on a large scale.

\section{Improved Clinical Metrics Following Implementation}

This series represents our efforts to utilize a combination of several techniques and technologies to achieve a more rapid and painless recovery after lumbar spinal fusion surgery. Using just 3 simple interventions (liposomal bupivacaine injection at the incision site, a single dose of intravenous acetaminophen at the end of surgery, and daily rounds by a medical student) resulted in the substantial clinical benefit. On all metrics, including medication consumption, narcotics usage per day, total narcotics usage, pain scores, mobility as measured by ambulation, and LOS, the ERAS patients performed better than historical controls.

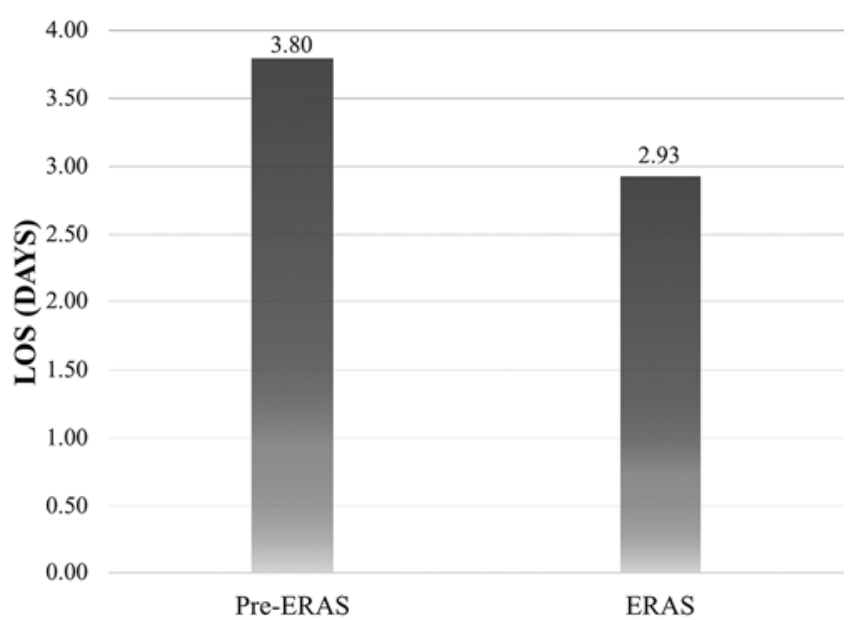

FIG. 5. Average hospital LOS for ERAS and pre-ERAS groups.

Taken as a whole, the reduction in narcotics usage, improved pain scores, greater ambulation, and faster discharge all indicate as a whole that the patients experienced reduced discomfort after surgery. If we had seen reduced narcotics usage but higher pain scores, then one could more likely attribute the effects of our program through an enforced protocol with limited benefits. Similarly, improved pain control with reduced ambulation could be seen as the result of an improved pain control program due to pharmacological adjustments, but with limited overall benefit.

\section{Generalizability Across Surgeon Participants}

We have previously published on technique-specific methods for reducing the burden of recovery following spinal fusion surgery.9.15,16 However, a key tenet of ERAS programs remains the ability to scale to implementation across institutions. Therefore, for this project 3 spinal neurosurgeons were involved equally. The surgeons implemented no change in their practice. Patient care did not deviate from their standard and practice with regard to patient selection, choice of operative technique, and surgical strategy employed.

\section{Study Limitations}

This study has significant limitations. First, as a singlecenter study, the results have to be validated across diverse practice environments and geographies. Second, as with all ERAS programs, the quality improvement program is not a randomized controlled trial. There are inherent differences between the historical and intervention cohorts. However, this is a consistent feature across ERAS platforms, and randomized programs are often neither feasible nor desirable given their tight selection criteria and thus lack of relevance to clinical practice. Third, some of the processes implemented are not available in all centers or nations, as 2 of our interventions include a pharmaceutical product not available in many countries. Finally, the results may not apply to surgeries excluded from this report, such as front/back spinal surgeries, longer-segment operations, and decompression operations without fusion. 


\section{Upcoming Iterative Improvements (versions 2.0 and 3.0)}

As with all institutions with ERAS programs, our institution is committed to a continuous quality improvement process. While we have seen substantial effects with version 1.0, we have already planned the next 2 iterative changes, with intervals to obtain data and inform ourselves longitudinally. For version 2.0, which will be launched in early 2019, we will incorporate 3 more interventions: 1 ) use of an educational video before surgery; 2) "pre-habilitation" planning, with goal setting and preparations for after care following discharge; and 3) a single dose of $600 \mathrm{mg}$ of gabapentin orally before surgery. Version 3.0 will include 1) carbohydrate nutrition 2 hours before surgery; 2) standardized pain medication protocols after surgery; and 3) twice-daily physical/occupational therapy after surgery.

\section{Conclusions}

The initial results in our ERAS program have been very promising in achieving its intended goals. The effects are likely transferrable to other institutions and have been achieved with little cost and minimal disruption to our standard practices. We anticipate further improvements with later iterations of our ERAS program.

\section{References}

1. Ali ZS, Ma TS, Ozturk AK, Malhotra NR, Schuster JM, Marcotte PJ, et al: Pre-optimization of spinal surgery patients: development of a neurosurgical enhanced recovery after surgery (ERAS) protocol. Clin Neurol Neurosurg 164:142-153, 2018

2. Grasu RM, Cata JP, Dang AQ, Tatsui CE, Rhines LD, Hagan $\mathrm{KB}$, et al: Implementation of an Enhanced Recovery After Spine Surgery program at a large cancer center: a preliminary analysis. J Neurosurg Spine 29:588-598, 2018

3. Kahokehr A, Sammour T, Zargar-Shoshtari K, Thompson L, Hill AG: Implementation of ERAS and how to overcome the barriers. Int J Surg 7:16-19, 2009

4. Kehlet H: Multimodal approach to control postoperative pathophysiology and rehabilitation. Br J Anaesth 78:606617, 1997

5. Kehlet H: Multimodal approach to postoperative recovery. Curr Opin Crit Care 15:355-358, 2009

6. Kehlet H, Dahl JB: The value of "multimodal" or "balanced analgesia" in postoperative pain treatment. Anesth Analg 77:1048-1056, 1993

7. Kehlet H, Wilmore DW: Multimodal strategies to improve surgical outcome. Am J Surg 183:630-641, 2002

8. Mathiesen O, Dahl B, Thomsen BA, Kitter B, Sonne N, Dahl $\mathrm{JB}$, et al: A comprehensive multimodal pain treatment reduces opioid consumption after multilevel spine surgery. Eur Spine J 22:2089-2096, 2013
9. Parker SL, Mendenhall SK, Shau DN, Zuckerman SL, Godil SS, Cheng JS, et al: Minimally invasive versus open transforaminal lumbar interbody fusion for degenerative spondylolisthesis: comparative effectiveness and cost-utility analysis. World Neurosurg 82:230-238, 2014

10. Pearson AM, Lurie JD, Tosteson TD, Zhao W, Abdu WA, Weinstein JN: Who should undergo surgery for degenerative spondylolisthesis? Treatment effect predictors in SPORT. Spine (Phila Pa 1976) 38:1799-1811, 2013

11. Singh K, Vaccaro AR, Kim J, Lorenz EP, Lim TH, An HS: Biomechanical comparison of cervical spine reconstructive techniques after a multilevel corpectomy of the cervical spine. Spine (Phila Pa 1976) 28:2352-2358, 2003

12. Soffin EM, Vaishnav AS, Wetmore D, Barber L, Hill P, Gang $\mathrm{CH}$, et al: Design and implementation of an Enhanced Recovery After Surgery (ERAS) program for minimally invasive lumbar decompression spine surgery: initial experience. Spine (Phila Pa 1976) [epub ahead of print], 2018

13. Wainwright TW, Immins T, Middleton RG: Enhanced recovery after surgery (ERAS) and its applicability for major spine surgery. Best Pract Res Clin Anaesthesiol 30:91-102, 2016

14. Wang MY, Chang PY, Grossman J: Development of an Enhanced Recovery After Surgery (ERAS) approach for lumbar spinal fusion. J Neurosurg Spine 26:411-418, 2017

15. Wang MY, Cummock MD, Yu Y, Trivedi RA: An analysis of the differences in the acute hospitalization charges following minimally invasive versus open posterior lumbar interbody fusion. Presented at the 2009 Joint Spine Section Meeting. J Neurosurg Spine 12:694-699, 2010

16. Wang MY, Lerner J, Lesko J, McGirt MJ: Acute hospital costs after minimally invasive versus open lumbar interbody fusion: data from a US national database with 6106 patients. J Spinal Disord Tech 25:324-328, 2012

\section{Disclosures}

Dr. Levi: honorarium from AANS, and grant support from the Department of Defense and National Institutes of Health (NINDS). Dr. Wang: consultant for DePuy-Synthes Spine, K2M, Stryker, and Spineology; patient holder with DePuy-Synthes Spine; and direct stock ownership in ISD.

\section{Author Contributions}

Conception and design: Wang, Levi, Manzano. Acquisition of data: Wang, Brusko, Kolcun, Heger, Madhavan, Urakov. Analysis and interpretation of data: Brusko, Levi, Manzano, Madhavan, Urakov, Epstein. Drafting the article: Wang, Brusko, Kolcun. Critically revising the article: Epstein. Statistical analysis: Brusko.

\section{Correspondence}

Michael Y. Wang: University of Miami Miller School of Medicine, Miami, FL. mwang2@med.miami.edu. 